\title{
ATUAÇÃO DE ENFERMEIROS NO COMBATE À COVID-19 NO ESTADO DA PARAÍBA: UM RELATO DE EXPERIÊNCIA
}

\section{ARTIGO ORIGINAL}

SANTOS, Genilson Gaudêncio dos ${ }^{1}$

JALES, Karla Michella Sobreira ${ }^{2}$

SANTOS, Edileide Alves dos ${ }^{3}$

SANTOS, Raquel Pereira dos ${ }^{4}$

OLIVEIRA, Josefa Juciélia Andrade de ${ }^{5}$

TORRES, Ester Gomes ${ }^{6}$

SANTOS, Genilson Gaudêncio dos. Et al. Atuação de enfermeiros no combate à COVID-19 no Estado da Paraíba: Um relato de experiência. Revista Científica Multidisciplinar Núcleo do Conhecimento. Ano 05, Ed. 11, Vol. 10, pp. 05-15.

${ }_{1}^{1}$ Pós-Graduado em Enfermagem do Trabalho pela Universidade Cândido Mendes UCAM; Pós-Graduado em Enfermagem de Urgência e Emergência pela Faculdade Integrada de Araguatins - FAIARA; Graduado em Enfermagem pela UNESC.

${ }^{2}$ Graduada em Enfermagem pela Faculdade Maurício de Nassau.

${ }^{3}$ Graduada em Enfermagem pela União de Ensino Superior de Campina Grande UNESC.

${ }^{4}$ Graduada em Enfermagem pela Faculdade Maurício de Nassau.

${ }^{5}$ Graduada em Enfermagem pela União de Ensino Superior de Campina Grande UNESC.

${ }^{6}$ Pós-Graduada em Enfermagem do Trabalho pelo Instituto Gianna Bereta. PósGraduada em Saúde do Adulto pelo Grupo FAVENNI. Bacharel em Enfermagem pelo Instituto Florence de Ensino Superior. 
Novembro de 2020. ISSN: 2448-0959, Link de acesso:https://www.nucleodoconhecimento.com.br/saude/atuacao-de-enfermeiros

\section{RESUMO}

Este artigo apresenta o relato da experiência da atuação de enfermeiros no combate à COVID-19 no Estado da Paraíba, ocorrido no período de maio a agosto de 2020. É Produto da atuação de enfermeiros na linha de frente em atendimento à necessidade excepcional e emergencial de combate à pandemia do novo coronavírus. O propósito é relatar à vivência de enfermeiros na assistência de enfermagem a pacientes portadores da doença, descrever os desafios enfrentados e êxitos obtidos, visando à valorização da enfermagem na assistência de pacientes críticos portadores da COVID-19 atendidos na atenção especializada. Concluímos que há a necessidade de as universidades e faculdades darem ênfase à competência profissional para lidar com a morte. Percebemos ainda, o quanto necessitamos ser preparados nesse sentido, sendo necessário uma abordagem além do técnico-científico (necessitando uma visão do humano). Abordaremos também, essa temática do ponto de vista biopsicoespiritual. Muitos foram os desafios superados durante a caminhada na luta contra o coronavírus. Favorecendo grande avanço pessoal e profissional, além de estreitar laços de cooperação com profissionais de diversas áreas mantendo uma interação multidisciplinar e interdisciplinar para o desenvolvimento de práticas e seguimentos da formação profissional.

Palavras-chave: COVID-19, assistência de enfermagem.

\section{INTRODUÇÃO}

No final do ano de 2019, na cidade de Wuhan na China começaram a surgir inúmeros casos de pneumonia, que mais tarde chamaram a atenção do mundo para essa nova doença. Daí em diante tem se discutido muito sobre o novo coronavírus (SARS-CoV2) e a doença que ele provoca. 
De acordo com o World Health Organization (2020) atualmente existem casos confirmados da COVID-19 em todos os continentes. Os termos oficiais referentes ao novo coronavírus e a COVID-19 foram anunciados pela Organização Mundial de Saúde em 11 de fevereiro de 2020, definidos da seguinte forma: Doença - COVID-19 (do inglês "Coronavírus Disease" 2019); Vírus - SARS-CoV-2 (do inglês "Severe Acute Respiratory Syndrome Coronavirus 2").

De acordo com Brasil (2020), embora a maioria das pessoas acometidas pelo COVID19 apresentem sintomas leves ou não complicados, algumas desenvolvem doença grave que requer oxigenoterapia (14\%), e aproximadamente 5\% necessitarão de tratamento em Unidade de Terapia Intensiva (UTI). Dentre os doentes críticos, a maioria necessitará de ventilação mecânica.

Com o surgimento de inúmeros questionamentos, da avalanche de informações sobre o assunto e da escassez de produção científica, enfatizamos a necessidade tão importante de uma enfermagem bem informada e preparada para atuar no atendimento dos novos casos da doença na assistência especializada e em todos os níveis de atenção de forma segura.

Ao longo deste artigo procuramos descrever sobre nossa vivência, experiência profissional, desafios, medos e êxitos diante desse novo cenário de pandemia que o mundo enfrenta.

Discorreremos também sobre importantes orientações aos profissionais de enfermagem acerca da assistência ao paciente crítico portador da COVID-19, abordando os conceitos que são dados ao novo coronavírus (SARS-CoV-2) e a doença COVID-19.

Conforme Brasil (2020), os primeiros vírus do tipo coronavírus em humanos foram isolados pela primeira vez em 1937. Mas somente em 1965 o vírus foi descrito como coronavírus, por conta de suas características peculiares, identificada à microscopia, em forma de coroa. 
Isso significa dizer que o coronavírus é um tipo de vírus que já existia há algum tempo, desde os anos 60, e ao longo da história têm aparecido de forma diferente causando prejuízos maiores principalmente para os homens.

Esse novo coronavírus difere um pouco dos outros que já existiram. Ele surgiu causando grande preocupação, devido ao fato de se espalhar rápido, podendo causar doença grave em algumas pessoas.

Para o atendimento à necessidade excepcional e emergencial de combate ao COVID19 no Estado da Paraíba, o governo lançou um edital para contratação de enfermeiros a fim de atuarem no combate do novo coronavírus (SARS-CoV-2) durante a pandemia.

Após todo o processo de inscrição e seleção dos candidatos, recebemos a chamada pública no diário oficial do Estado da Paraíba, a qual prontamente atendemos e iniciamos nossas atividades.

No primeiro momento participamos de um treinamento profissional que foi uma experiência riquíssima do ponto de vista profissional, pois, fomos acolhidos, informados sobre como seria nossa vivência nos recintos e no ambiente profissional do Pronto Atendimento do COVID-19 (PACOVID-19), que passamos a chamar de Ala Covid.

Durante o treinamento recebemos todas as instruções de paramentação e desparamentação, quanto ao uso de EPl's necessários no exercício da função, ou seja, na atuação do enfermeiro dentro da ALA COVID.

No início do mês de Setembro 2020 a ALA COVID do Hospital de Emergência e Trauma de Campina Grande foi desativada devido à redução de casos da COVID-19 no Estado da Paraíba. A ALA COVID continha 60 leitos, sendo 30 leitos de Unidade de Terapia Intensiva (UTI) e 30 leitos de enfermaria destinados ao atendimento à pessoa portadora do coronavírus. 
O Hospital de Emergência e Trauma de Campina Grande, após o fechamento da UTI e da Enfermaria Covid, informou que houve 371 atendimentos, 250 altas hospitalares e 40 intubações com sucesso.

De acordo com a UNASUS - UFMA (2020) a oferta de informações à população apresenta-se como estratégia no controle da transmissão do vírus, na medida em que o usuário é responsável por identificar sinais e sintomas que acusam a possível infecção e/ou o agravamento do quadro clínico, nos casos suspeitos de COVID-19, possibilitando a procura por unidades de saúde de forma oportuna.

\section{METODOLOGIA}

Este estudo consistiu em um relato de experiência que descreveu a vivência dos autores, na oportunidade de um contrato de prestação de serviço, por prazo de 90 dias, ocorrido no período de maio a agosto de 2020, firmado com o Governo do Estado da Paraíba. Trata-se de um olhar qualitativo, que abordou a vivência e a assistência de enfermagem à pacientes portadores da COVID-19 a partir de método descritivo.

O relato de experiência é um método de pesquisa descritiva que traz uma reflexão sobre certa atuação ou ação que aborda uma situação vivenciada no âmbito profissional de interesse da comunidade científica.

A experiência profissional que resultou na redação deste relato aconteceu no Hospital de Emergência e Trauma Dom Luiz Gonzaga Fernandes situado na cidade de Campina Grande no Estado da Paraíba.

Utilizou-se das seguintes técnicas para a redação do relato: diário profissional com rodas de conversa profissional, (pesquisador participante), participação nas atividades clínicas/assistenciais. Não foram utilizados dados pessoais de pacientes e de profissionais neste estudo.

\section{RESULTADOS E DISCUSSÕES}




\section{ASSISTÊNCIA DE ENFERMAGEM AO PACIENTE CRÍTICO PORTADOR DA COVID-19}

Os enfermeiros profissionais que foram contratados para exercer sua função na Ala Covid do Hospital de Emergência e Trauma Dom Luiz Gonzaga Fernandes, iniciaram suas atividades no mês de maio de 2020 até agosto de 2020. A Ala Covid teve como foco o tratamento de pacientes portadores da COVID-19 provenientes de todos os municípios do Estado da Paraíba. Esta se dividia em enfermaria e UTI-COVID.

O enfermeiro é o líder responsável pela equipe de enfermagem e pela assistência conferida ao paciente. São eles que realizam a consulta de enfermagem com histórico de anamnese, exame físico contendo os sinais vitais e realizam a Sistematização da Assistência de Enfermagem (SAE).

A assistência de enfermagem era voltada ao paciente crítico portador da COVID-19 apresentando função respiratória comprometida como principal sintoma relacionado à COVID-19. Outros sintomas identificados foram: dispnéia, desconforto respiratório, dessaturação, hipertermia, hipoxemia, hemodinâmica instável, intubados em uso de drogas vasoativas entre outros.

A assistência prezava atender as necessidades humanas em todos os níveis de complexidade (desde o básico até as necessidades mais complexas) dos pacientes internados na Unidade de Terapia Intensiva (UTI) prezamos por uma assistência humanizada conferida pelo o uso da Sistematização da Assistência de Enfermagem (SAE) observando todas as suas etapas:

- Histórico de Enfermagem

- Diagnóstico de Enfermagem

- Planejamento de Enfermagem

- Prescrição de Enfermagem

- Evolução ou Avaliação de Enfermagem 
O Histórico de Enfermagem conforme Carvalho (2001) é uma maneira de realizar o levantamento de dados sobre o paciente, família e comunidade e pode ser dividido em três partes - a identificação, anamnese e exame físico.

Os Diagnósticos de Enfermagem conforme Carpenito apud Tannure; Gonçalves (2008) baseiam-se tanto nos problemas reais (voltados para o presente) quanto nos problemas potenciais (voltados para o futuro), que podem ser sintomas de disfunções fisiológicas, comportamentais, psicossociais ou espirituais.

O Planejamento de Enfermagem para Bachion apud Tannure; Gonçalves (2008) consiste em um plano de ação para alcançar objetivos e resultados em relação a um diagnóstico de enfermagem.

A Prescrição de Enfermagem conforme Carvalho (2001) é uma ordem do enfermeiro para sua equipe com o objetivo de direcionar a assistência, orientando toda a equipe sobre o que fazer, quando e como realizar a assistência.

A Evolução ou Avaliação de Enfermagem de acordo com Tannure; Gonçalves (2008) consiste na ação de acompanhar as respostas do paciente à assistência prescrita, por meio de registro nos prontuários ou locais próprios, é nessa etapa que o enfermeiro avalia as melhoras do paciente, inclui medidas corretivas e, se necessário, revê a prescrição de enfermagem.

Na UTI COVID e em toda a Ala Covid era aplicada à metodologia da Sistematização da Assistência de enfermagem para todos os pacientes portadores da COVID-19. Os registros da assistência de enfermagem e da (SAE) eram digitalizados no prontuário eletrônico do paciente (prontuário computadorizado), e impresso em papel para ser anexado ao prontuário físico de cada paciente. Neste eram colocados também os registros dos demais profissionais da equipe multidisciplinar, a saber; médicos, fisioterapeutas, nutricionistas, odontólogos, fonoaudiólogos entre outros.

Com frequência nossos pacientes estavam intubados e recebendo ventilação mecânica invasiva. Nosso olhar profissional exigia constantemente muita precisão 
com o exame físico, sinais e sintomas, e com as técnicas de monitoração. Devíamos sempre estar atentos a possível lesão pulmonar induzida por ventilador mecânico.

Por se tratar de pacientes intubados observamos o tempo todo se havia sincronia do paciente e do ventilador mecânico, bem como, agitação, inquietação e outros sinais de desconforto respiratório (uso excessivo dos músculos intercostais e acessórios da respiração, movimentos descoordenados do tórax e abdome e se o paciente se queixa de falta de ar).

Havendo alguns desses sintomas supracitados o enfermeiro responsável pelo paciente anotava qualquer alteração nos sinais vitais e as evidência de instabilidade hemodinâmica, relatando-as para o médico responsável imediatamente.

Atentamos sempre para esses sinais e sintomas por que eles indicam se o paciente estava deteriorando ou se a ventilação mecânica era ineficaz.

Os pacientes críticos portadores da COVID-19 por estarem intubados e sedados, recebiam alimentação enteral, portanto, o enfermeiro deveria sempre ajustar e inspecionar se a cabeceira do leito estava elevada para evitar possível broncoaspiração.

$\mathrm{Na}$ assistência de enfermagem ao paciente crítico portador da COVID-19 o enfermeiro faz a ausculta pulmonar anterior e posterior utilizando-se dos métodos da ausculta, percussão e palpação do tórax, identificando os sons da respiração normal e de possíveis ruídos adventícios. Os métodos propedêuticos são indispensáveis na assistência de enfermagem ao paciente criticamente doente, entubado ou não.

Já o método da percussão do tórax foi realiado para avaliação na evidência de derrame pleural; por que na evidência do mesmo, a região pulmonar acometida é maciça à percussão e nessa mesma área os barulhos respiratórios ficam ausentes.

Aprendemos que a inobservância da ausculta das áreas dependentes dos pulmões dos pacientes resulta em despercepção pelo enfermeiro de eventos como a atelectasia ou derrame pleural que pode estar acontecendo. 
O enfermeiro é também o profissional responsável pela coleta e execução (realização) do exame de gasometria arterial. O exame resulta da punção de uma artéria (radial ou femoral) no caso dos pacientes críticos portadores da COVID-19, este exame é feito rotineiramente de modo que os pacientes conscientes quando submetidos a essa monitorização gasométrica constante e rotineira relatam dor considerável, por isso é imprescindível o enfermeiro explicar a importância que esse exame tem para condução do seu tratamento.

Diante de pacientes conscientes portadores da COVID-19, a abordagem do enfermeiro no tocante a parte humanizada e psicológica de acolhimento, conferindo segurança e confiança ao paciente é imprescindível, diante dos sentimentos de medo da morte. Nessa situação específica se faz necessário que o enfermeiro informe e/ou solicite a presença do psicólogo para o acompanhamento de casos específicos.

\section{DESAFIOS SUPERADOS DURANTE A CAMINHADA}

Para nós era uma experiência nova mistificada pelo medo e a insegurança, tivemos de trabalhar nossos medos, incertezas e sentimentos. Muitos de nós precisamos recorrer ao serviço de assistência e suporte psicológico do próprio hospital que disponibilizavaum atendimento específico para os profissionais da Ala Covid.

Tivemos de superar o medo de nos contaminarmos com o vírus, uma possibilidade e uma fatalidade muito eminente. E se contaminados ficaríamos bem? Como reagiria nosso organismo ao vírus? Convivemos com notícias constantes de colegas nossos que foram contaminados.

Éramos constantemente bombardeados pelas notícias de quantos colegas, enfermeiros, médicos que perderam a vida atuando na linha de frente no combate do coronavírus. Muitos de nós fomos contaminados pelo vírus. Aqueles que foram contaminados receberam assistência médica na própria Ala Covid onde obtiveram atestado médico para afastamento das atividades, tratamento e repouso. 
O medo constante de nos contaminarmos e/ou contaminarmos nossos familiares era constante, por esse motivo, muitos de nós nos distanciamos (nos isolamos) de nossas famílias por prevenção daqueles a quem amamos, o que foi muito difícil para nós que somos pais, mães, irmãos e filhos.

A enfermagem é a profissão da área de saúde que mais tem contato físico e mais passa tempo junto do paciente, portanto, sabíamos que éramos os mais propensos à contaminação e ao adoecimento.

Esta é a ciência do cuidado do ser humano, nosso objeto de estudo e trabalho. Somos nós, os enfermeiros, que também conferimos o último cuidado ao ser humano, que é o cuidado ao corpo pós-morte (atividade realizada pela equipe de enfermagem com muita ética e respeito ao corpo pós-morte).

Também foi um grande desafio lidar com o fato de que os pacientes não poderiam ter seus familiares por perto como acompanhantes, isto gerou uma nova demanda para nós profissionais de enfermagem, que tivemos de suprir também esse papel de apoio emocional exercido pela família dos pacientes, tentando esmaecer suas ansiedades, angústias, medo diante da doença e da morte.

Essa foi uma demanda muito grande no nosso campo emocional e psicológico, tivemos que estreitar laços de cooperação com profissionais de diversas áreas, mantendo uma interação multidisciplinar para o desenvolvimento de práticas e seguimentos da formação profissional.

Ainda não existem informações fidedignas sobre a história natural da COVID-19, nem condutas efetivas para o tratamento clínico das infecções por coronavirus em seres humanos. Sabemos que o vírus tem transmissibilidade alta, que causa uma síndrome respiratória aguda, podendo esta ser leve ou grave.

Lidamos com o sentimento constante de impotência diante da morte e do morrer de nossos pacientes. Esse enfrentamento foi díficil para muitos de nós, por que a finitude humana é uma etapa crucial da existência, tanto para o ser humano quanto para nós, enfermeiros profissionais, que evidenciamos tão cruel realidade. 
O processo de finitude, morte, e a comunicação da notícia da morte pela equipe de saúde aos familiares das vítimas, era uma constante demanda emocional que exigia maior competência psicológica da equipe multidisciplinar. Enfatizo que a equipe multidisciplinar de profissionais precisa estar preparada para esses acontecimentos, diante do luto, da morte e do morrer.

Diante dessa situação tivemos que compreender o que é o cuidado paliativo no seu mais amplo aspecto, bem como, o processo da morte e do morrer, o significado do luto $e$ as medidas de intervenção junto a ele. Adquirimos habilidades para compreender o papel da enfermagem e da equipe interdisciplinar diante de pacientes críticos portadores da COVID-19 no tocante aos cuidados paliativos, a morte, o morrer, o luto enfrentado pelos familiares.

A assistência de enfermagem aos pacientes críticos ou não portadores da COVID-19 não teve somente a parte do luto e das perdas, mas, tiveram também nossos êxitos e méritos, primeiro por ter salvado inúmeras vidas, devolvendo as famílias muitos de nossos pacientes recuperados desta doença.

Segundo, por termos nos reinventado enquanto profissionais de saúde que tiveram coragem de atender ao tão necessário chamado para combater a pandemia do SARSCoV-2 e com muito zelo e maestria prestaram a assistência mais digna aos pacientes que necessitaram do nosso cuidado.

O Hospital de Emergência e Trauma de Campina Grande ao fechamento da UTI e da Enfermaria COVID informou que houve 371 atendimentos, 250 altas hospitalares e 40 intubações com sucesso.

\section{CONSIDERAÇÕES FINAIS}

À conclusão deste relato, podemos dizer que há uma necessidade de que a academia, as universidades e faculdades dêem ênfase à competência profissional para lidar com a morte. 
Percebemos quanto ainda necessitamos ser preparados e adquirir competências emocionais nesse sentido, sendo necessária uma abordagem além do técnicocientífico, (necessitando uma visão mais do humano) abordando essa temática do ponto de vista biopsicoespiritual.

Muitos foram os desafios superados durante a caminhada na luta contra o coronavírus, favorecendo grande avanço pessoal e profissional, além de estreitar laços de cooperação com profissionais de diversas áreas mantendo uma interação multidisciplinar para o desenvolvimento de práticas e seguimento da formação profissional.

Até o presente não existe medicamento exato para o tratamento da COVID-19 e nem uma vacina. Porém é necessário considerar as demais patologias de base que os pacientes acometidos pelo coronavírus são portadores, com o objetivo de oferecer ações assistenciais de cuidado, tratamento e suporte para o manejo clínico da doença.

Considerando o poder de transmissão e a rápida disseminação do novo coronavírus, medidas de prevenção e proteção tem que ser intensificadas para conter o significativo aumento de casos da COVID-19.

Nessa situação de pandemia vivenciada é importante que os enfermeiros e demais profissionais eduquem a população e enfatizem sobre as medidas de prevenção e proteção que envolvem o isolamento social e comportamentos de higiene respiratória, pessoal e comunitária, pois, elas são as medidas mais necessárias e eficientes para que a disseminação e transmissão do SARS-CoV-2 seja controlada e diminuída.

Toda informação acerca da nova doença (COVID-19) e sobre o seu agente etiológico o SARS-CoV-2 são pontos importantes a serem conhecidos por toda a enfermagem e demais profissionais, em se tratando principalmente de uma doença recentemente descoberta.

Neste relato de experiência procuramos traçar uma linha tênue sobre alguns aspectos da vivência e da assistência de enfermagem ao paciente crítico portador da COVID- 
19, bem como, a importância da atuação do enfermeiro no combate a pandemia que vivenciamos no mundo inteiro do novo coronavírus.

Em relação a assistência de enfermagem aos pacientes, críticos ou não, portadores da COVID-19 não tivemos somente a parte do luto e das perdas, mas, tivemos também nossos êxitos e méritos, primeiro por termos salvado inúmeras vidas, devolvendo às suas famílias muitos de nossos pacientes recuperados da doença provocada pelo novo coronavírus.

Segundo, por termos nos reinventado enquanto profissionais de saúde que tiveram coragem de atender ao tão necessário chamado para combater a pandemia do SARSCoV-2 e com muito zelo e maestria prestaram a assistência mais digna aos pacientes que necessitam do nosso cuidado.

O Hospital de Emergência e Trauma de Campina Grande ao fechamento da UTI e da Enfermaria covid informou que houve 371 atendimentos, 250 altas hospitalares e 40 intubações com sucesso.

\section{REFERÊNCIAS}

BRASIL. MINISTÉRIO DA SAÚDE. O que é coronavírus? (COVID-19). Brasília: Ministério da Saúde, 2020.

\section{BRASIL. MINISTÉRIO DA SAÚDE. Secretaria de Atenção Especializada à Saúde.} Departamento de Atenção Hospitalar, Domiciliar e de Urgência. Protocolo de manejo clínico da Covid-19 na Atenção Especializada [recurso eletrônico] / Ministério da Saúde, Secretaria de Atenção Especializada à Saúde, Departamento de Atenção Hospitalar, Domiciliar e de Urgência. - 1. ed. rev. - Brasília: Ministério da Saúde, 2020.

CARVALHO, G. M. Enfermagem do Trabalho. São Paulo: EPU,2001.

TANNURE, M. C.; GONÇALVES, A. M. P. Sistematização da Assistência de Enfermagem. Rio de Janeiro: Guanabara Koogan, 2008. Cap. 1, p. 7 - 19. 
UFMA. Curso Orientações gerais ao paciente com COVID-19 na Atenção Primária à Saúde. São Luís: março, 2020.

UNIVERSIDADE ABERTA DO SUS - UFMA. Orientações aos usuários sobre as manifestações clínicas e condições de risco. In: UNIVERSIDADE ABERTA DO SUS

WORLD HEALTH ORGANIZATION. Novel Coronavirus (COVID-19) Situation. 2020. Disponível em: https://who.maps.arcgis.com/ Acesso em: 08 set. 2020.

Enviado: Setembro, 2020.

Aprovado: Novembro, 2020. 